\title{
Desarrollo de Procedimientos Normalizados de Trabajo: una forma Innovadora de realizar las Prácticas en Asignaturas de Ciencias Experimentales
}

\author{
Valeria Gallo, Rafael Beltrán, Dolores Hernanz y Ana Sayago \\ Departamento de Química y Ciencia de los Materiales, Área de Química Analítica, Facultad de \\ ciencias Experimentales, Universidad de Huelva, Avd. Tres de Marzo S/N. 21071, Huelva-España \\ (e-mail: ana.sayago@dqcm.uhu.es)
}

Recibido Feb. 25, 2011; Aceptado Mar. 29, 2011; Versión final recibida May. 02, 2011

\begin{abstract}
Resumen
En este trabajo se plantea al alumno el aprendizaje autónomo mediante la elaboración de procedimientos normalizados de trabajo (PNTs). La experiencia se realiza en la asignatura Métodos Analíticos de Control de Calidad del plan de Ingeniería Química y de Ingeniería Técnica Industrial especialidad Química de la Universidad de Huelva en España. La elaboración de los PNTs e instrucciones de trabajo facilitan que las tareas repetitivas se realicen siempre de la misma forma con independencia de la persona que las realiza, constituyendo una buena herramienta para la formación práctica del alumnado. El grado de satisfacción del alumno para el aprendizaje autónomo ha sido evaluado a través de entrevistas personales, tutorías grupales y encuestas. Los resultados han sido muy satisfactorios y la actividad ha resultado ser un importante agente motivador del aprendizaje.
\end{abstract}

Palabras clave: procedimientos normalizados de trabajo, aprendizaje autónomo, motivación, calidad de la enseñanza

\section{Development of Standard Operating Protocols: an Innovative way to the Practice of the course Analytical Methods of Quality Control}

\begin{abstract}
The present work proposes the use of autonomous learning for students through the elaboration of standard operating procedures (SOPs). This experience is carried out within the course Analytical Methods for Quality Assurance of the majors Chemical Engineering and Technical Industrial Engineering in Chemistry at the University of Huelva in Spain. The preparation of the SOPs and the working instructions make that repetitive actions are performed in the same way regardless of the person who performs the tasks. Therefore, SOPs represent a good tool in the practical formation of students. The degree of satisfaction of the students in using this method of autonomous learning has been evaluated through individual interviews, group tutorial and opinion polls. The results have been satisfactory and the activity has resulted to be a motivating agent for the learning process.
\end{abstract}

Keywords: standard operating procedures, self-learning, motivation, quality of teaching 


\section{INTRODUCCIÓN}

Desde la Convergencia Europea se nos propone un cambio de la conceptualización del modelo de Universidad. Esta innovación afecta al estilo docente del profesorado universitario que debe adaptarse a las nuevas exigencias. Debe haber una preocupación constante, por parte de las instituciones educativas y por parte de los docentes, en redefinir la didáctica de las clases, pues no se trata solamente de enseñar contenidos heterogéneos a partir de experiencias y estudios fragmentados sino de posibilitar la asociación de la teoría con la práctica y la motivación del aprendizaje (Petitat, 1994; Maceiras et al., 2010).

Los conceptos y estrategias, definidos en la Declaración de Bolonia (1999) para la construcción de un EEES, suponen un cambio en los programas educativos que deben adaptarse a un nuevo proceso formativo basado en dos puntos fundamentales: 1) aprendizaje de conocimientos específicos propios del título y 2) desarrollo de las habilidades y destrezas necesarias para adaptar dichos conocimientos a un campo profesional. Por tales motivos, se pretende que el alumnado además de adquirir los conceptos, se familiarice con el uso de recursos y herramientas imprescindibles para los futuros egresados ya que la sociedad actual se caracteriza por una cantidad de información infinita, dinámica y cambiante, un extensivo uso de Internet y las nuevas tecnologías, y un mercado laboral en continuo cambio, lo que requiere profesionales no sólo con conocimientos específicos y básicos, sino con destrezas para aplicarlos y resolver los problemas de un modo creativo, implicando de este modo un aprendizaje autónomo y durante toda la vida, y capacidad para trabajar en grupos multidisciplinares (Dochy et al., 2003; Ribeiro y Mizukami, 2005).

La calidad, es hoy día, un recurso estratégico para la competitividad, con una gran incidencia en numerosos ámbitos profesionales, productivos y sociales. En una sociedad en la que existe una amplia oferta, la Calidad ha dejado de ser una ventaja competitiva para convertirse en una exigencia del mercado. Los laboratorios analíticos no son una excepción, y si bien el concepto de calidad de la medida ha estado siempre implícito en la química analítica, ha sido en los últimos años cuando se ha producido un planteamiento explícito y sistemático de la calidad en los laboratorios analíticos, derivados del concepto de "garantía de calidad", siendo esta una herramienta esencial para asegurar los resultados analíticos (Rauf et al, 2009). En las buenas prácticas de laboratorio se definen los PNTs como aquellos documentos que describen como realizar ensayos o actividades normalmente no detalladas en los protocolos o guías. La norma UNE-EN-ISO 9000 (UNE 2000a) define un procedimiento como una forma específica de llevar a cabo una actividad o un proceso, estos procedimientos son un componente vital en cualquier sistema de gestión de calidad (Hattemer-Apostel, 2001).

El cumplimiento de las buenas prácticas de laboratorio constituye un requisito indispensable para garantizar la calidad e integridad de los resultados analíticos. La elaboración de PNTs e instrucciones de trabajo posibilitan que las tareas repetitivas se realicen siempre de la misma forma independientemente de la persona que las realiza, además toda persona involucrada en estas tareas a través de los PNTs posea la información necesaria para un correcto proceder, sabiendo en todo momento lo que tiene que hacer, cómo y cuándo. Basándonos en la importancia que esto puede tener para los futuros profesionales no sólo a nivel de conocimientos sino de competencias, el planteamiento principal de este trabajo, ha sido trasladar a los laboratorios de prácticas, el concepto de Sistema de Gestión de la Calidad bajo los principios de Buenas Prácticas de Laboratorio (BPL), a través de la elaboración de PNTs.

\section{METODOLOGÍA}

Para el desarrollo del proyecto se plantearon dos objetivos generales. Como primer objetivo se buscó implicar directamente al alumnado en el aprendizaje teórico-práctico de la asignatura Métodos Analíticos de Control de Calidad, concretamente en la elaboración de PNTs como material didáctico que podrá ser utilizado además de para su propia formación, para la de cualquier alumno que realice asignaturas de carácter práctico. Todo ello de acuerdo con la filosofía de trabajo propuesto por los nuevos planes de estudio según el Espacio Europeo de 
Enseñanza Superior (EEES). El segundo objetivo, consistió en estimular la participación de los alumnos en el desarrollo de la asignatura, y así conseguir una mayor motivación del alumnado, que sirva como refuerzo positivo para el estudio práctico de la asignatura.

Para el desarrollo y cumplimiento de los objetivos propuestos, el equipo docente participante del proyecto, seleccionó inicialmente los equipos de laboratorio para los que se realizarían los Procedimientos Normalizados de Trabajo. El buen funcionamiento de todos los instrumentos es esencial para la obtención de datos válidos y de calidad, haciendo hincapié en la necesidad de la calibración de los equipos, en la necesidad de garantizar la representatividad estadística de los resultados y en el análisis de errores. El equipo docente responsable de las asignaturas, presentaron al inicio de las clases el proyecto a desarrollar. Para fomentar la participación del alumnado en éste, se les informo de su carácter como actividad académica dirigida y por tanto evaluable. Se realizaron equipos de trabajo, cada uno de los cuales eligió un equipo de laboratorio (balanza analítica, material volumétrico, micropipetas, termómetros, espectrofotómetros) para desarrollar el PNT.

La acción tutorial tiene una gran relevancia dentro del nuevo marco del aprendizaje fundamentada en el campo de la función del docente como guía-asesor-transmisor (Michavila y Zamorano, 2007; Benito y Cruz, 2005). Por ello, se dedicaron varias de las sesiones presenciales a la organización de tutorías grupales. En estas tutorías se resolvieron dudas sobre la temática del trabajo y el alcance del mismo, se orientó al alumnado sobre los recursos bibliográficos en la Web, enseñándole a realizar búsquedas científicas y se ayudó al alumno en la selección del material más adecuado para el desarrollo de los PNTs. Una vez aclaradas todas las dudas, cada grupo de trabajo elaboró su protocolo y se evaluaron los resultados a través de entrevistas personales, tutorías grupales y encuestas.

\section{RESULTADOS Y DISCUSIÓN}

\section{Grado de cumplimiento de los objetivos}

En cuanto al grado de cumplimiento de los objetivos establecidos, se podría destacar el aprendizaje activo y cooperativo que ha supuesto la interacción entre los alumnos. Se ha potenciado en ellos la adquisición de distintas competencias como el trabajo en equipo, la escucha activa, la capacidad de crítica y autocrítica y la capacidad de síntesis. Además, el desarrollo de procedimientos ha fomentado los hábitos necesarios para llevar a cabo cualquier sistema de gestión de calidad. La realización de este proyecto ha permitido un mayor contacto del profesorado con el alumnado y ha constituido una buena ocasión para completar la formación de los profesores implicados. Por un lado, ha supuesto un mayor conocimiento del alumnado y ha permitido ayudar a reflexionar sobre la metodología más adecuada para conseguir los objetivos propuestos. Ha supuesto un reto para el equipo docente intentar conseguir al alumno investigador, buscador y constructor del conocimiento. Por otro lado en este proyecto, los profesores involucrados también han tenido que adaptarse a trabajar en equipo, para actuar como un equipo docente.

La elaboración de material didáctico ha servido para que el alumno adquiera el conocimiento necesario a la hora de realizar búsquedas bibliográficas (Web, libros, revistas científicas) y sepa seleccionar la información necesaria (capacidad crítica), además de adquirir habilidades de redacción y de síntesis. Para la elaboración del mismo, se debatió en clase la forma más adecuada de presentarlo y finalmente los profesores presentaron un guión orientador (Fig. 1) que fue distribuido a todos los estudiantes para que permitiese obtener un formato homogéneo en la totalidad del proyecto. Se elaboraron PNTs sobre el funcionamiento y calibración de la balanza granatario y balanza analítica, calibración de material volumétrico, almacenamiento de reactivos, calibración de termómetros, funcionamiento de un espectrofotómetro Ultravioleta-Visible y funcionamiento y calibración de un $\mathrm{pH}$-metro, cada uno de los cuales fue realizado por un equipo de trabajo. Los alumnos participaron en la corrección de los PNTs a través de las tutorías realizadas con cada equipo en particular, las cuales también fueron utilizadas para evaluar el alcance del aprendizaje con esta nueva metodología. En general, ha supuesto una experiencia 
muy satisfactoria de intercambio de conocimientos y el grado de consecución fue adecuado a los objetivos previstos.

\section{Evaluación de los resultados del proyecto.}

Como principal resultado de este proyecto, podría destacarse el elevado grado de motivación e implicación que han mostrado los alumnos en su desarrollo. La satisfacción de los alumnos se ha ido poniendo de manifiesto durante las tutorías y en las clases presenciales.

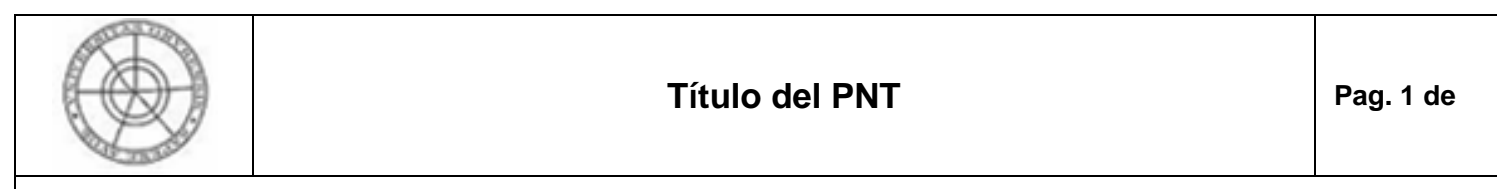

1. OBJETIVOS.-

Definir claramente el objeto del PNT, es decir, especificar la metodología de trabajo empleada, por ejemplo: definir el sistema, definir los criterios.

\section{ALCANCE.-}

Se establecerá el campo de aplicación del PNT, teniendo en cuenta que existen PNT comunes y particulares de cada laboratorio, además existen PNT válidos para diversos equipos, materiales, que posee una gran semejanza en su uso, aplicación o realización de los mismos.

\section{REFERENCIAS.-}

Se indicarán los documentos utilizados como referencia para realizar el PNT, así como otros documentos que lo complemente.

Se redactará, siempre que sea aplicable, agrupando las referencias en los siguientes subapartados:

\subsection{DOCUMENTOS UTILIZADOS EN LA ELABORACIÓN DEL PNT.-}

En todos los casos se hará referencia a la norma (UNE-EN ISO/IEC 17025)

Además, se referenciarán todos aquellos documentos externos (normas, reglamentos, manuales) que hayan sido utilizados como referencia, o que se hubiera utilizado/consultado durante la elaboración del PNT, indicando su revisión.

\subsection{DOCUMENTOS A UTILIZAR CONJUNTAMENTE CON ESTE PNT.-}

Se indicarán todos aquellos documentos internos y/o externos que se deben utilizar conjuntamente con el PNT, para desarrollar la actividad o actividades en él descrita.

\section{GENERAL.-}

Cuando sea necesario, este apartado detallará toda aquella información que se considere útil y/o ayude a la utilización y compresión del PNT. Por ejemplo: definiciones, abreviaturas, antecedentes, introducción teórica, siempre que proceda en éste PNT.

\section{DESCRIPCIÓN/REALIZACIÓN.-}

Detallará la secuencia de actuación en la actividad objeto del PNT, medios necesarios, responsabilidades, registros generados, criterios de aceptación o rechazo, si procede.

Por tanto es la parte gruesa del PNT, donde se describe todos y cada uno de los métodos, equipos, materiales, así como el uso, secuencia y modo de empleo y realización de los mismos para el análisis y obtención del PNT.

En general varía en función del PNT, por tanto, algunos son obvios y necesarios, y otros se omiten al no proceder en este tipo de PNT (dependiendo del tipo de PNT, se utilizarán otras estrategias de realización o descripción), por ejemplo: periodicidad, material y equipos empleados, preparación, realización (explicación secuencial y temporal del procedimiento), tratamiento de los resultados (obtención de resultados y recogidos en tablas). "Existen numerosas formas de describir y realizar el proceso, usad las más viables y convenientes según cada tipo de PNT".

\section{ANEXOS.-}

Contendrán todos aquellos documentos, tablas, planos, fotos, gráficas, que se consideren necesarios para usar, interpretar y evaluar la utilización del PNT.

Fig. 1: Formato y guión orientador para la elaboración de los PNTs 
Se ha aplicado un cuestionario al finalizar el proyecto donde se ha podido comprobar la satisfacción del alumnado en diversos conceptos. El cuestionario ha sido realizado por la totalidad de los alumnos participantes del proyecto, treinta y dos entre las dos asignaturas. La figura 2 muestra el cuestionario realizado a los alumnos así como la valoración promedio obtenida para las mismas. Cada ítem fue valorado en una escala de uno a cuatro.

\section{ENCUESTA DE SATISFACCIÓN}

1. Cuando el profesor presentó el proyecto a desarrollar me pareció una forma muy interesante de llevar a cabo el trabajo práctico de la asignatura

2. Los objetivos del proyecto han sido definidos claramente por el profesor.

3. La actuación del profesor ha aumentado mi interés por la asignatura.

4. Mi interés por el trabajo ha ido aumentando a lo largo de su desarrollo.

5. Se proporcionaron las fuentes para la búsqueda de la información básica.

6. La bibliografía y el material sugerido han ayudado a la preparación y la realización de los PNTs.

7. El tamaño del grupo permitió el aprovechamiento del trabajo en equipo

8. Mis compañeros/as me han ayudado a realizar mi trabajo

9. La realización del proyecto favorece el desarrollo de mi capacidad para trabajar en equipo

10. La elaboración de PNTs favorece el desarrollo de mi capacidad para analizar y resolver problemas

11. La realización del proyecto favorece la aplicación de conocimientos

12. La realización del proyecto favorece el desarrollo mi sentido crítico

13. Las actividades propuestas favorecen el aprendizaje autónomo

14. La realización del proyecto me ha ayudado a la comprensión de la materia

15. En general estoy satisfecho con los trabajos desarrollados.

16. El ambiente de trabajo era agradable

17. El docente tiene ha tenido actitud receptiva y amistosa con los alumnos.

18. El docente se ha preocupado de que los estudiantes aprendan.

19. El docente ha estado disponible para las consultas de los alumnos durante las tutorías.

20. En general, estoy satisfecho con el trabajo llevado a cabo por el profesor.

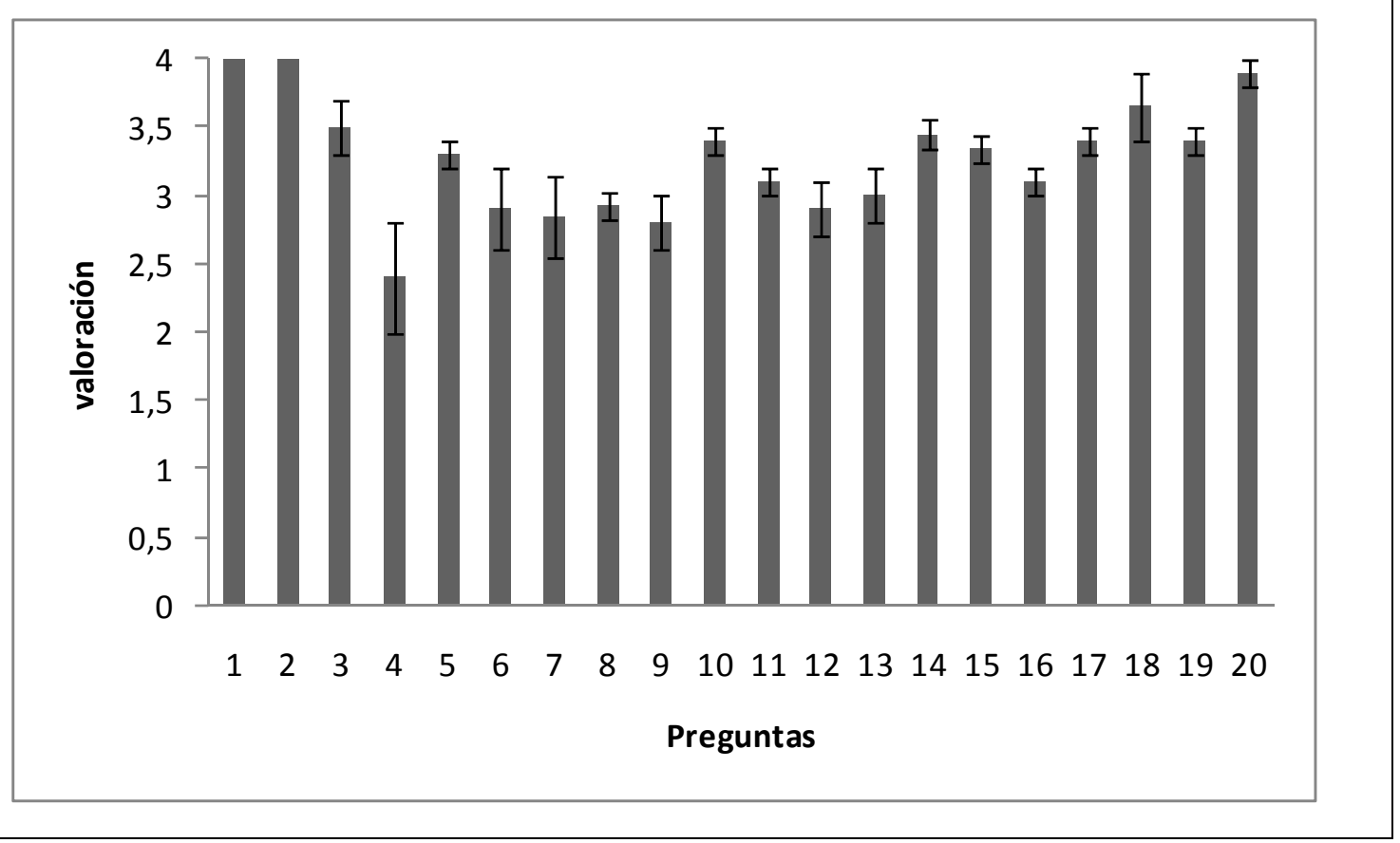

Fig. 2: Encuesta de satisfacción realizada y análisis de los resultados. Valoración: 1=nada; 2=poco; 3=bastante; 4=mucho 


\section{CONCLUSIONES}

Este proyecto de innovación docente, nos ha permitido durante su desarrollo introducirnos en el concepto del proceso de enseñanza-aprendizaje, conceptos planteados en los acuerdos de Bolonia.

Se han aplicado propuestas formadoras centradas en el trabajo del alumno, como son el aprendizaje colaborativo y el papel del profesor como orientador o ayuda a la actividad de aprendizaje del alumno.

El Proyecto se ha aplicado a dos asignaturas optativas que se imparten en los últimos años de las titulaciones y los alumnos han sido capaces de afianzar/consolidar muchas capacidades y competencias que habían adquirido a lo largo de su experiencia universitaria. Se ha manifestado claramente que el aprendizaje y el contenido interesan más cuando hay implicación personal y cuando es activo, es por ello que los resultados alcanzados han sido muy satisfactorios. La actividad ha resultado ser un importante agente motivador del aprendizaje.

\section{AGRADECIMIENTOS}

Los miembros del equipo docente agradecen al servicio de Innovación Docente de la Universidad de Huelva, la oportunidad que les ha brindado al concederles este proyecto.

\section{REFERENCIAS}

Benito A. y Cruz, A., Nuevas claves para la Docencia Universitaria en la Espacio Europeo de Educación Superior, $1^{\mathrm{a}}$ ed, Narcea S.A. de Ediciones, Madrid, España (2005).

Cooper M., Greenbow, T. Pienta, N., Chemists' guide to effective teaching, Prentice Hall, Upper Saddle River, New Jersey (2009).

Declaración de Bolonia. Declaración conjunta de los ministros europeos de educación reunidos en Bolonia el 19 de junio de 1999. Bolonia, (1999).

Dochy, F., Segers, M., Van den Bossche, P., Gijbels, D., Effects of problem-based learning: a metaanalysis, Learning and Instruction: 13(5), 533-568 (2003).

Hattemer-Apostel, R. Standard operating procedures - a novel perspective. The Quality assurance Journal 5(4), 207-219 (2001).

Michavila, F., Zamorano, S., Reflexiones sobre los cambios metodológicos anunciados en la Educación Superior en España, Educación y Futuro: 16, 31-46 (2007).

Maceiras, R., Cancelas, A., Goyanes, V., Aplicación de Nuevas Tecnologías en la Docencia Universitaria, Formación Universitaria, 3 (1), 21-26 (2010).

Petitat, A. Produção da escola. Produção da sociedade. pp 122 Ed. Artes Medicas, Porto Alegre, Brasil. (1994).

Rauf, M. A., Hanan, A. Quality assurance considerations in chemical analysis. The Quality assurance Journal 12(1), 16-21 (2009).

Ribeiro, L.R., Mizukami, M.G., Problem-based learning: a student evaluation of an implementation in postgraduate engineering education, European J. Eng. Educ.: 30(1), 137-149 (2005). 